\title{
Finite Time Prescribed Performance Control for Uncertain Second-Order Nonlinear Systems
}

\author{
Hui Lv, ${ }^{1,2}$ Wei Xiang $\mathbb{D}^{2}{ }^{2}$ and Jun Zhu $\mathbb{D}^{1,3}$ \\ ${ }^{1}$ Key Laboratory of Mathematical Modelling and High Performance Computing of Air Vehicles (NUAA), MIIT, \\ Nanjing University of Aeronautics and Astronautics, Nanjing 210016, Jiangsu, China \\ ${ }^{2}$ School of Finance and Mathematics, Huainan Normal University, Huainan 232038, China \\ ${ }^{3}$ State Key Laboratory of Mechanics and Control of Mechanical Structures, Nanjing University of Aeronautics and Astronautics, \\ Nanjing 210016, Jiangsu, China
}

Correspondence should be addressed to Jun Zhu; zhujun@nuaa.edu.cn

Received 30 November 2021; Accepted 18 January 2022; Published 16 February 2022

Academic Editor: Peijun Wang

Copyright $(2022$ Hui Lv et al. This is an open access article distributed under the Creative Commons Attribution License, which permits unrestricted use, distribution, and reproduction in any medium, provided the original work is properly cited.

In this article, we discuss the finite time stability problem for second-order systems with an uncertain nonlinear function. A finite time performance function with the sinusoidal function is constructed, and the constrained problem of the original system is transformed into the stability problem of the equivalent system. Combining prescribed performance control and fuzzy logic systems, an effective control method is proposed. The simulation results also prove that the method we adopted is effective.

\section{Introduction}

Second-order nonlinear systems [1-6] have a variety of potentials in the real world, such as the robotic manipulator, horizontal platform system, navigation system, and gyro system. At present, many control methods [6-14] have been proposed for second-order nonlinear systems. For example, Zhao et al. [7] developed an output feedback control method for the second-order nonlinear system with uncertain parameters. For uncertain second-order nonlinear systems, a saturated controller was proposed in [8], which can overcome the system uncertainty and external disturbance. In [9], an adaptive fuzzy distributed control scheme was proposed, which realized the consensus tracking consistent of the second-order multiagent system. An adaptive PID control method was developed in [10], which makes the output error of the system tend to zero and ensures the stability of the system. In order to deal with the fault regulation problem of a class of second-order nonlinear systems, Van [11] proposed a high-order terminal sliding mode control method. It should be pointed out that the above control methods can only ensure that the tracking error enters a small neighborhood of zero, but the neighborhood and the time to reach the neighborhood cannot be set in advance.

Recently, many researchers have been studying the prescribed performance control (PPC) method [12-18]. The idea is to transform the restriction problem of an original system into the stability problem of an equivalent system by using the performance function and transformation function. However, the traditional PPC method cannot solve the presetting time problem. Therefore, in recent years, different types of finite time performance functions were proposed. In [19], a finite time performance function with the exponential function was proposed, and the designed PPC method realized that the tracking error converges to the predefined zone in presetting time. Using the same finite time performance function in [19], Tran and Ho [20] studied the PPC strategy of uncertain horizontal platform system. In [21], a finite time performance function was constructed by polynomials, based on the partial persistent excitation condition, the tracking error of the strict feedback system can approach the predefined zone in presetting time and unknown functions can also be estimated accurately.

Inspired by the above work, this paper will construct a finite time performance function through the sinusoidal 
function to investigate the stability of uncertain secondorder nonlinear systems. The overall structure of this paper is as follows. In Section 2, some preliminaries are presented. The finite time PPC method and its stability analysis are investigated in Section 3. Section 4 gives an example for simulation. Finally, the conclusion is given in Section 5 .

\section{System Descriptions and Problem Formulations}

Consider the following uncertain second-order nonlinear system:

$$
\ddot{x}(t)=f(t, x)+g(t, x) u(t),
$$

where $x(t) \in \mathbb{R}$ is the system state, $x=[x(t), \dot{x}(t)]^{T}, f(t, x)$ is an unknown nonlinear function, and $u(t) \in \mathbb{R}$ is the control input. Let $x_{d}(t) \in \mathbb{R}$ be a desired signal. The following assumptions are provided for later discussion.

Assumption 1. States $x(t), \dot{x}(t), x_{d}(t), \dot{x}_{d}(t)$, and $\ddot{x}_{d}(t)$ are measurable.

Assumption 2. The nonlinear function $f(t, x)$ is unknown but bounded.

Assumption 3. $g(t, x)$ is known, and $g(t, x) \neq 0$ for all $t \geq 0$ and $x$.

Define the tracking error $e(t)=x(t)-x_{d}(t)$; the aim of this paper is to limit the tracking error $e(t)$ within the preset boundary through the finite time prescribed performance control method.

In order to make the tracking error $e(t)$ meet the steadystate performance and transient performance, the following constraint condition is designed:

$$
-\rho(t)<e(t)<\rho(t)
$$

where $\rho(t)$ is a finite time performance function and defined as

$$
\rho(t)= \begin{cases}a_{0} \sin ^{3}\left(\frac{\pi}{2 T}(T-t)\right)+a_{1}, & 0 \leq t \leq T, \\ a_{1}, & T<t,\end{cases}
$$

where $a_{0}$ and $a_{1}$ are preset positive parameters and $T$ is the preset time. Usually, the tracking error $e$ is transformed into an equivalent expression using the transformation function. In this article, the transformation function is defined as follows:

$$
\Gamma(z)=\frac{e^{z}-e^{-z}}{e^{z}+e^{-z}}
$$

Define $e(t)=\rho(t) \Gamma(z)$; if $z(t)$ is bounded, then $\Gamma(z)$ satisfies $-1<\Gamma(z)<1$, which implies that the tracking error $e(t)$ is limited within $(-\rho(t), \rho(t))$. Therefore, the following work focuses on the boundedness of $z(t)$.
Remark 1. Similar to performance function (3), we can also use the cosine function to design a finite time performance function as

$$
\rho(t)= \begin{cases}a_{0} \cos ^{3}\left(\frac{\pi}{2 T}(T-t)\right)+a_{1}, & 0 \leq t \leq T, \\ a_{1}, & T<t,\end{cases}
$$

where parameters $\alpha_{0}$ and $\alpha_{1}$ are the same as those in (3).

Remark 2. The design of the transformation function is also important for the PPC method, and transformation function (4) can also be changed as

$$
z=\frac{e(t)}{p(t)}
$$

In order to ensure $|z|<1$, the construction of the barrier Lyapunov function needs to be considered.

\section{Control Design and Stability Analysis}

Now, the first derivative of $e(t)$ can be obtained as

$$
\dot{e}(t)=\dot{\rho}(t) \Gamma(z)+\rho(t) \frac{\partial \Gamma(z)}{\partial z} \dot{z}(t)
$$

which, together with (7), one gets

$$
\dot{z}(t)=\Pi_{1}(t)+\Lambda(t)\left(\dot{x}(t)-\dot{x}_{d}(t)\right),
$$

where $\quad \Pi_{1}(t)=-(\dot{\rho}(t) \Gamma(z) / \quad \rho(t)(\partial \Gamma(z) / \partial z)), \Lambda(t)=$ $(1 / \rho(t)(\partial \Gamma(z) / \partial z))$. According to (3) and (4), one has

$$
0<a_{1} \leq \rho(t) \leq \rho(0), 0<\frac{\partial \Gamma(z)}{\partial z}=\frac{4}{\left(e^{z}-e^{-z}\right)^{2}} \leq 1 .
$$

So, $\Lambda(t)$ satisfies the inequality $\Lambda(t) \geq(1 / \rho(0))>0$. From (7), one gets

$$
\begin{aligned}
\ddot{e}(t)= & \ddot{\rho}(t) \Gamma(z)+2 \dot{\rho}(t) \frac{\partial \Gamma(z)}{\partial z} \dot{z}(t)+\rho(t) \frac{\partial^{2} \Gamma(z)}{\partial z^{2}}(\dot{z}(t))^{2} \\
& +\rho(t) \frac{\partial \Gamma(z)}{\partial z} \ddot{z}(t) .
\end{aligned}
$$

Combining (1), (7), and (10), one obtains

$$
\ddot{z}(t)=\Pi_{2}(t)+\Lambda(t)\left(f(t, x)+g(t, x) u(t)-\ddot{x}_{d}(t)\right),
$$

where $\quad \Pi_{2}(t)=-(\ddot{\rho}(t) \Gamma(z)+2 \dot{\rho}(t)(\partial \Gamma(z) / \partial z) \dot{z}(t)+\rho$ $\left.(t)\left(\partial^{2} \Gamma(z) / \partial z^{2}\right)(\dot{z}(t))^{2} / \rho(t)(\partial \Gamma(z) / \partial z)\right)$. In order to prove that $z(t)$ is bounded, we introduce a new variable $s(t)$ as

$$
s(t)=\dot{z}(t)+c_{1} z(t)
$$

where $c_{1}$ is a positive design parameter.

Since $f(t, x)$ is unknown, we will employ fuzzy logic systems to estimate $f(t, x)$ in system (1). According to Lemma 2 in [19], there exists a fuzzy logic system (FLS) $\theta^{* T} w(x)$ such that 


$$
f(t, x)=\boldsymbol{\theta}^{* T} w(x)+\varepsilon_{f}(x),
$$

where $\theta^{*}$ is the ideal constant weight vector, $w(x)$ is the basis function vector, and $\varepsilon_{f}(x)$ is the bounded approximation error, i.e., there exists positive constant $\overline{\varepsilon_{f}}$ such as $\left|\varepsilon_{f}(x)\right| \leq \overline{\varepsilon_{f}}$. Let $\widehat{f}(x, \widehat{\theta})=\widehat{\theta}^{T} w(x)$, where $\widehat{\theta}$ is the estimation of $\theta^{*}$ and $\theta=\theta^{*}-\widehat{\theta}$ is the estimation error. In this paper, the controller $u(t)$ is designed as

$$
u(t)=\frac{-\Lambda(t) \hat{\theta}^{T} w(x)-\Pi_{3}(t)-c_{2} s(t)}{\Lambda(t) g(t, x)},
$$

where $\Pi_{3}(t)=\Pi_{2}(t)-\Lambda(t) \ddot{x}_{d}(t)+c_{1}\left(\Pi_{1}(t)+\Lambda(t) \dot{x}(t)-\right.$ $\left.\Lambda(t) \dot{x}_{d}(t)\right)$ and $c_{2}$ is a positive constant. And choose the adaptive law of $\widehat{\theta}$ as

$$
\dot{\hat{\theta}}=\gamma_{f}\left(\Lambda(t) w(x) s(t)-\lambda_{f} \widehat{\theta}\right),
$$

where $\gamma_{f}>0$ and $\lambda_{f}>0$. Now, we give the main conclusions as follows.

Theorem 1. Under given Assumptions 1-3, when initial conditions $|x(0)|<\rho(0)$ are satisfied, then controller (14) and the parameter adaptive law (15) can guarantee that all signals of the closed-loop system are bounded, which also means that the tracking error $e(t)$ is limited within constraint condition (2).

Proof. Consider the Lyapunov function as follows:

$$
V(t)=\frac{1}{2}\left[s^{2}(t)+\frac{1}{\gamma_{f}} \tilde{\theta}^{T} \widetilde{\theta}\right] .
$$

By taking the derivative of $V(t)$, we can get

$$
\begin{aligned}
\dot{V}(t) & =s(t) \dot{\mathcal{s}}(t)+\frac{1}{\gamma_{f}} \tilde{\theta}^{T} \dot{\tilde{\theta}} \\
& =s(t)\left(\ddot{z}(t)+c_{1} \dot{z}(t)\right)+\frac{1}{\gamma_{f}} \tilde{\theta}^{T} \dot{\tilde{\theta}} \\
& =s(t)\left(\Pi_{3}(t)+\Lambda(t) f(t, x)+\Lambda(t) g(t, x) u(t)\right)-\frac{1}{\gamma_{f}} \tilde{\theta}^{T} \widehat{\theta} \\
& =s(t)\left(\Pi_{3}(t)+\Lambda(t) \boldsymbol{\theta}^{* T} w(x)+\Lambda(t) \varepsilon_{f}(x)+\Lambda(t) g(t, x(t)) u(t)\right)-\frac{1}{\gamma_{f}} \tilde{\theta}^{T} \widehat{\theta} .
\end{aligned}
$$

Substituting (14) and (15) into $\dot{V}(t)$ yields

$$
\begin{aligned}
\dot{V}(t)= & s(t)\left(\Lambda(t) \widetilde{\theta}^{T} w(x)+\Lambda(t) \varepsilon_{f}(x)-c_{2} s(t)\right) \\
& -\widetilde{\theta}^{T}\left(\Lambda(t) w(x) s(t)-\lambda_{f} \widehat{\theta}\right) \\
= & \Lambda(t) s(t) \varepsilon_{f}(x)-c_{2} s^{2}(t)+\lambda_{f} \widetilde{\theta}^{T} \widehat{\theta}
\end{aligned}
$$

Because the inequality described in the following holds,

$$
\begin{aligned}
\Lambda(t) s(t) \varepsilon_{f}(x) & \leq \frac{\Lambda^{2}(t)}{4} s^{2}(t)+\bar{\varepsilon}_{f}^{2}, \\
\lambda_{f} \widetilde{\theta}^{T} \widehat{\theta} & \leq-\frac{\lambda_{f}}{2} \widetilde{\theta}^{T} \widetilde{\theta}+\frac{\lambda_{f}}{2} \boldsymbol{\theta}^{* T} \boldsymbol{\theta}^{*} .
\end{aligned}
$$

Substituting the above inequalities into (18), one gets

$$
\dot{V}(t) \leq-\left(c_{2}-\frac{\Lambda^{2}(t)}{4}\right) s^{2}(t)-\frac{\lambda_{f}}{2} \widetilde{\boldsymbol{\theta}}^{T} \widetilde{\boldsymbol{\theta}}+\tau^{*},
$$

where $\tau^{*}=\bar{\varepsilon}_{f}^{2}+\left(\lambda_{f} / 2\right) \theta^{* T} \theta^{*}$, and select positive constant $c_{3}$ such that $c_{3}<\min \left\{2 c_{2}-\left(\Lambda^{2}(t) / 2\right), \gamma_{f} \lambda_{f}\right\}$. So, one has

$$
\dot{V}(t) \leq-c_{3} V(t)+\tau^{*},
$$

which implies that

$$
V(t) \leq \frac{\tau^{*}}{c_{3}}+\left(V(0)-\frac{\tau^{*}}{\dot{c}_{3}}\right) e^{-c_{3} t} \leq 2\left(V(0)+\frac{\tau^{*}}{c_{3}}\right) .
$$

Obviously, all signals in (16) are ultimately uniformly bounded. Assume that the bounded value of $s(t)$ is $\bar{s}$. Let $\bar{V}(t)=(1 / 2) z^{2}(t)$; according to (8), one has

$$
\begin{aligned}
\dot{\bar{V}}(t) & =z(t) \dot{z}(t) \\
& =z(t)\left(s(t)-c_{1} z(t)\right) \\
& =z(t) s(t)-c_{1} z^{2}(t) \\
& \leq-\left(c_{1}-\frac{1}{4}\right) z^{2}(t)+\bar{s}^{2} .
\end{aligned}
$$

Select parameter $c_{1}$ so that $c_{1}>(1 / 4)$, which means that $z(t)$ is bounded. Therefore, we can conclude that $e(t)$ is limited within $(-\rho(t), \rho(t))$. This completes the proof.

\section{Numerical Simulations}

In this part, an uncertain gyroscope system [22] is used to show the availability of the proposed method in this paper. The gyroscope system is described as

$$
\ddot{x}=f(t, x)+g(t, x) u(t),
$$




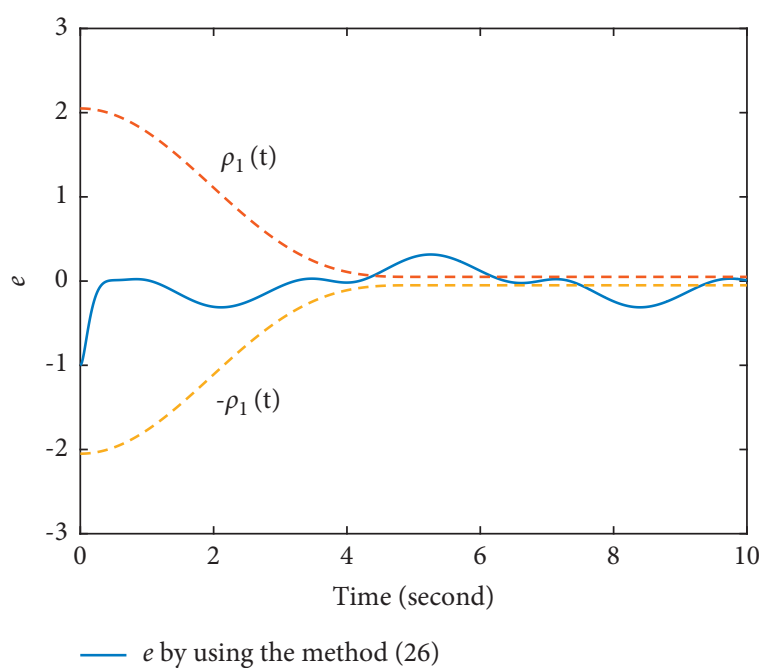

FIGURE 1: Time response trajectory of tracking error $e$ by using method (26).

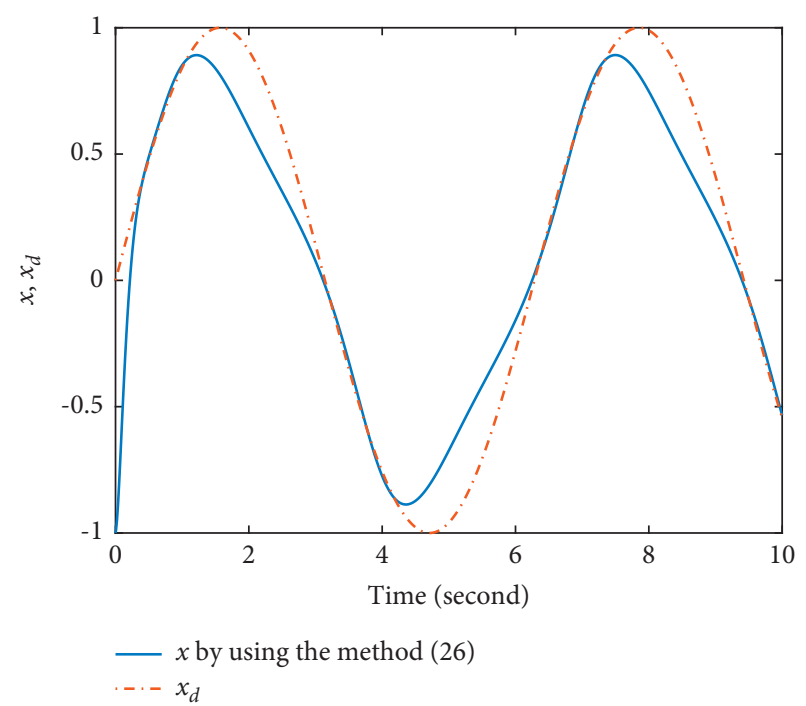

FIgURE 2: Time response trajectories of $x$ and $x_{d}$ by using method (26).

where $\quad x=[x, \dot{x}]^{T}, \quad f(t, x)=-A_{1}^{2}\left((1-\cos x)^{2} /\right.$ $\left.\sin ^{3} x\right)+A_{2} \sin x-A_{3} \dot{x}-A_{4} \dot{x}^{3}+A_{5} \sin (w t) \sin x$, and $g(t, x)=3+\sin x \sin \dot{x}$. Parameters $A_{1}, A_{2}, A_{3}, A_{4}, A_{5}$, and $w$ are selected as $A_{1}=10, A_{2}=1, A_{3}=0.5$, $A_{4}=0.05, A_{5}=35.7$, and $w=2$. The initial values of system (24) are $x(0)=-1, \dot{x}(0)=1$, and $x_{d}(t)=\sin (t)$. The fuzzy sets are defined over $[-5,5]$ for $x$ and $\dot{x}$. The corresponding fuzzy membership functions are chosen as follows:

$$
w(\xi)=\exp \left[-\frac{1}{2}\left(\frac{\xi+7.5-2.5 l}{1.2}\right)^{2}\right],
$$

where $\xi=x, \dot{x}$ and $l=1,2,3,4,5$. In order to compare with the proposed method in this paper, the traditional feedback method is designed as follows:

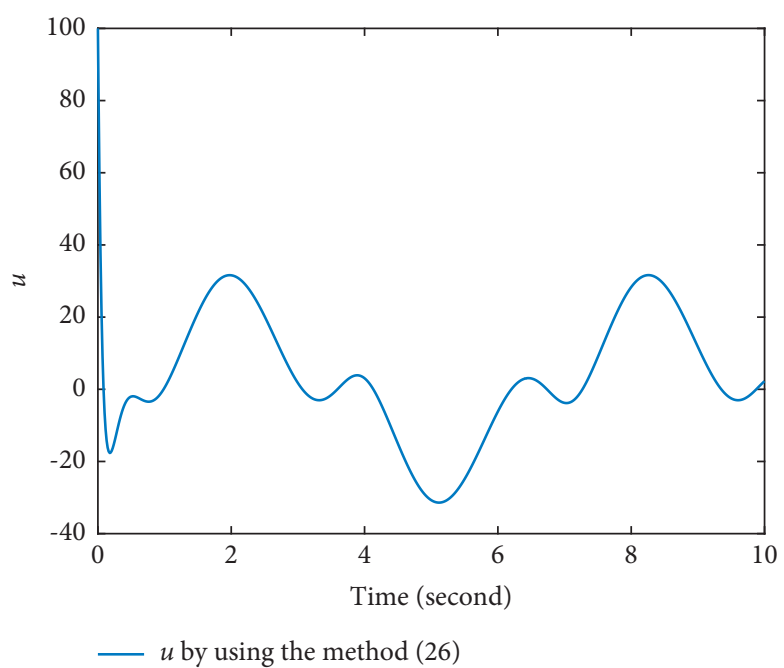

FIGURE 3: Time response trajectory of controller $u$ by using method (26).

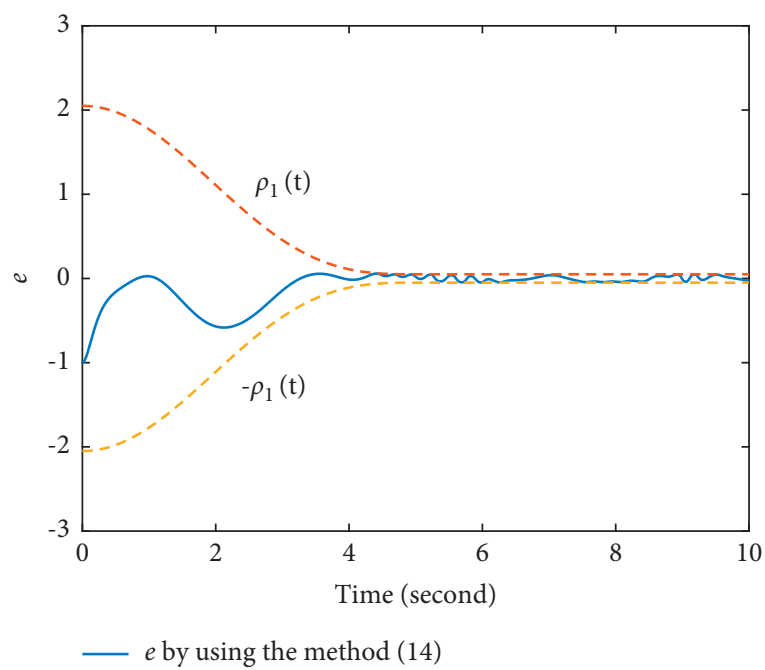

Figure 4: Time response trajectory of tracking error $e$ by using method (14) under $\rho_{1}(t)$.

$$
\left\{\begin{array}{l}
e(t)=x(t)-x_{d}(t), \\
\bar{s}(t)=\dot{e}(t)+c_{1} e(t), \\
u(t)=\frac{1}{g(t, x)}\left[-\widehat{\theta}^{T} w(x)+\ddot{x}_{d}(t)-c_{1} \dot{e}(t)-c_{2} \bar{s}(t)\right], \\
\dot{\hat{\theta}}=\gamma_{f}\left(w(x) \bar{s}(t)-\lambda_{f} \widehat{\theta}\right),
\end{array}\right.
$$

where parameters $c_{1}, c_{2}, \gamma_{f}$, and $\lambda_{f}$ are designed as $c_{1}=c_{2}=5, \gamma_{f}=3$, and $\lambda_{f}=0.05$. The control effect of traditional method (26) is shown in Figures 1-3. 


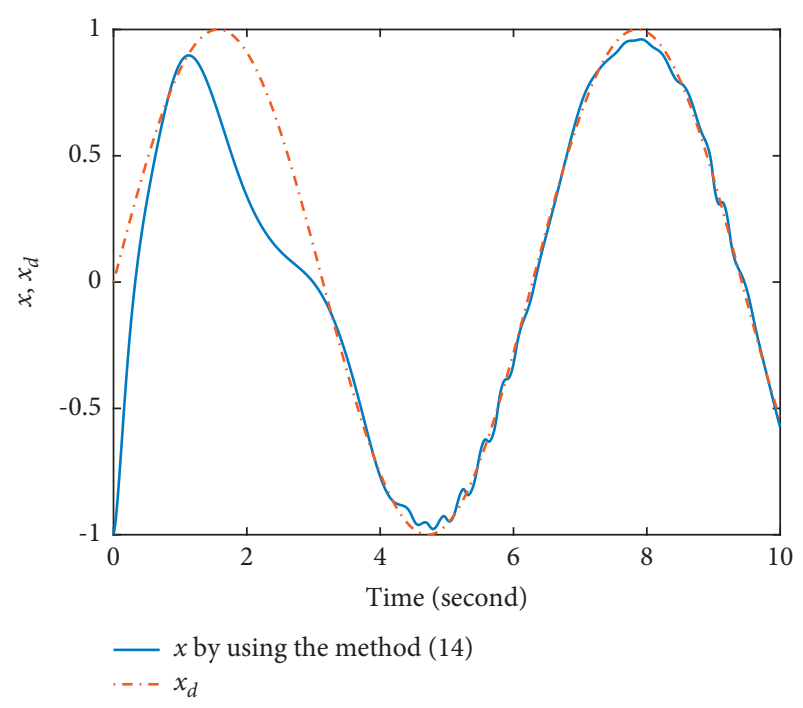

FIgURE 5: Time response trajectories of $x$ and $x_{d}$ by using method (14) under $\rho_{1}(t)$.

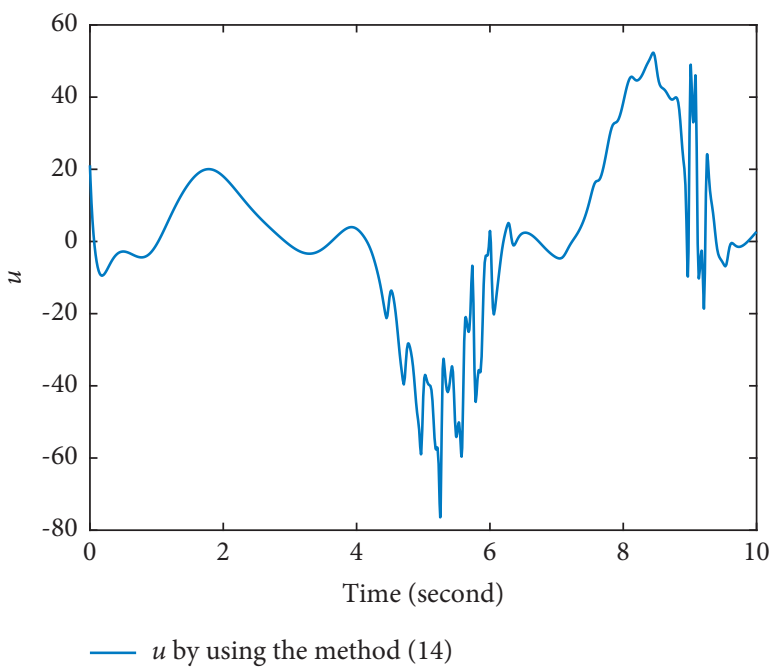

FIGURE 6: Time response trajectory of controller $u$ by using method (14) under $\rho_{1}(t)$.

Obviously, the tracking error $e(t)$ is not effectively controlled by using traditional method (26). With the above same parameters, the prescribed function $\rho(t)$ is designed as

$$
\rho_{1}(t)= \begin{cases}1.97 \sin ^{3}\left(\frac{(5-t) \pi}{10}\right)+0.05, & t \leq 5, \\ 0.05, & t>5 .\end{cases}
$$

The control effect of controller (14) is shown in Figures 4-6. It can be seen that the control effect of $e(t)$ has been improved, and the tracking error $e(t)$ has been limited within $[-0.05,0.05]$ after 5 seconds. One strong point of the

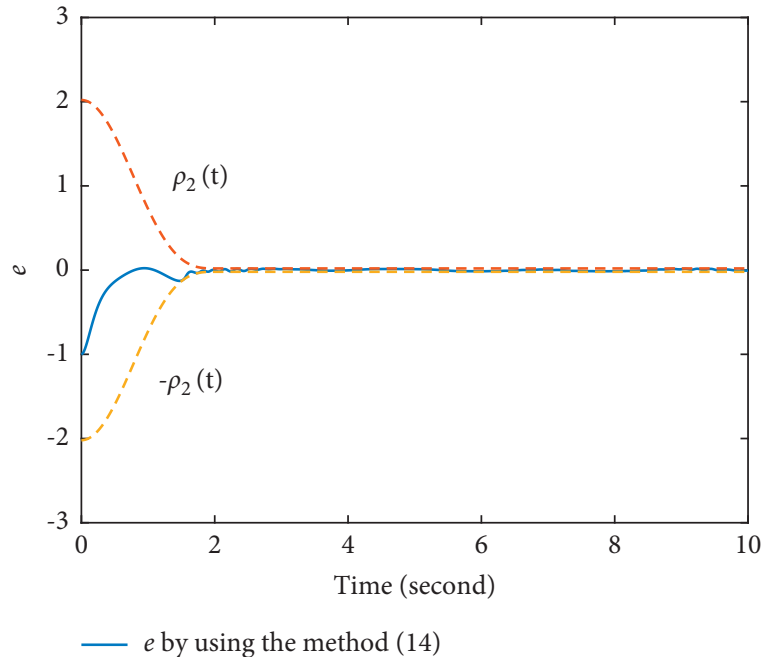

Figure 7: Time response trajectory of tracking error $e$ by using method (14) under $\rho_{2}(t)$

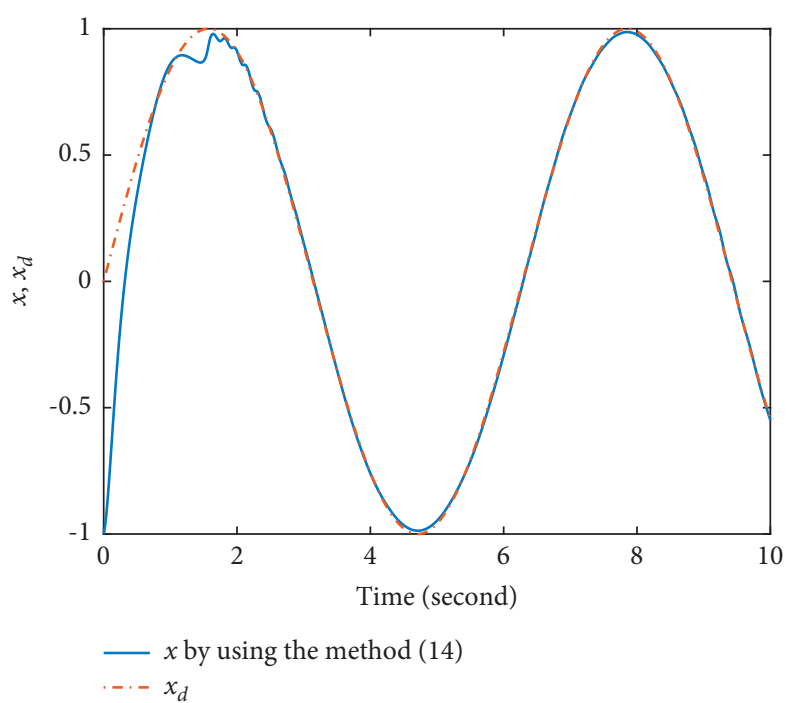

FIgURE 8: Time response trajectories of $x$ and $x_{d}$ by using method (14) under $\rho_{2}(t)$.

proposed method (14) is that it can change the control time and control interval, such as setting the prescribed performance function as

$$
\rho_{2}(t)= \begin{cases}1.97 \sin ^{3}\left(\frac{(3-t) \pi}{6}\right)+0.02, & t \leq 3, \\ 0.02, & t>3 .\end{cases}
$$

The control effect is shown in Figures 7-9. The above simulation results show that the proposed method (14) has better transient performance in practical applications. 


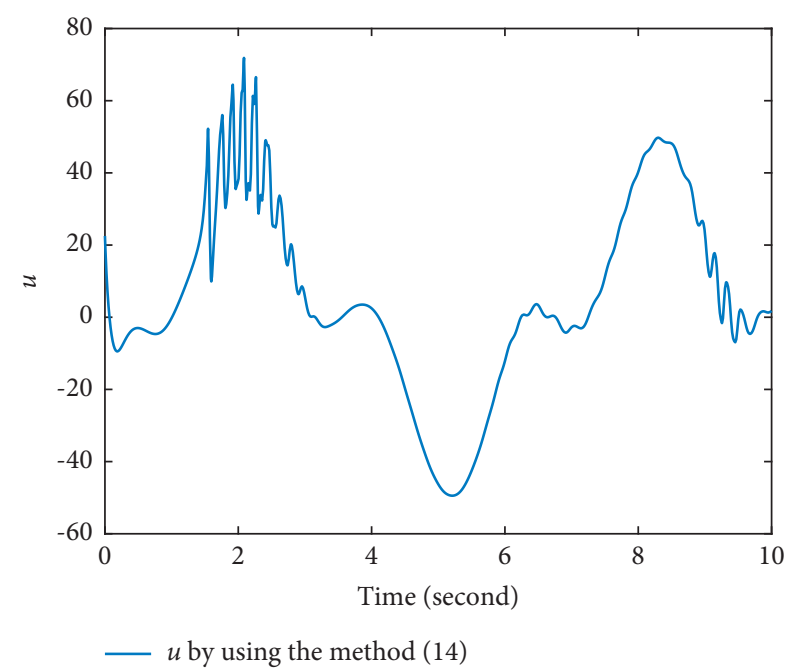

FIGURE 9: Time response trajectory of controller $u$ by using method (14) under $\rho_{2}(t)$.

\section{Conclusion}

We studied the finite time control problem of uncertain second-order nonlinear systems in this article. For the sake of the tracking error reaching the preset zone at the preset time, a finite time performance function was introduced. Meanwhile, the fuzzy logic system (FLS) was used to evaluate the uncertain function of the system. Through theoretical analysis, this paper proves that the proposed control method achieves the expected control effect. At the same time, this conclusion is also verified by simulation.

\section{Data Availability}

The data used in this paper are reflected in the manuscript.

\section{Conflicts of Interest}

The authors declare no conflicts of interest.

\section{Acknowledgments}

This research was supported by the NSFC Grant no. 11872210 and Grant no. MCMS-I-0120G01 and the Key Research Projects of Natural Science in Colleges and Universities of Anhui Province (KJ2019A0695, KJ2020A0644, and KJ2021A0965).

\section{References}

[1] R. Zhao, J. Yu, H. Yang, and Y.-H. Chen, "Contact constraints-based dynamic manipulation control of the multifingered hand robot: a force sensorless approach," Nonlinear Dynamics, vol. 107, no. 1, pp. 1081-1085, 2021.

[2] S. M. Amrr and A. Alturki, "Robust control design for an active magnetic bearing system using advanced adaptive SMC technique," IEEE Access, vol. 9, 2021.

[3] J. Liu and C. Li, "Match and mismatched second-order sliding mode finite-time control with simple parameter conditions and its applications," Journal of Control and Decision, pp. 112, 2021.

[4] L. Shi and M. Dang, "Design of manipulator control system of forest picking robot based on fractional order PID sliding mode control," Forest Chemicals Review, pp. 132-140, 2021.

[5] G. Bartolini, A. Ferrara, and E. Usai, "Output tracking control of uncertain nonlinear second-order systems," Automatica, vol. 33, no. 12, pp. 2203-2212, 1997.

[6] C. Zhao and L. Guo, "Pid controller design for second order nonlinear uncertain systems," Science China Information Sciences, vol. 60, no. 2, pp. 1-13, 2017.

[7] D. Zhao, S. K. Spurgeon, and X. Yan, "Adaptive output feedback finite time control for a class of second order nonlinear systems," in Proceedings of the 2016 14th International Workshop on Variable Structure Systems (VSS), pp. 53-58, IEEE, Nanjing, China, June 2016.

[8] N. Fischer, Z. Kan, R. Kamalapurkar, and W. E. Dixon, "Saturated rise feedback control for a class of second-order nonlinear systems," IEEE Transactions on Automatic Control, vol. 59, no. 4, pp. 1094-1099, 2013.

[9] C. P. Chen, C.-E. Ren, and T. Du, "Fuzzy observed-based adaptive consensus tracking control for second-order multiagent systems with heterogeneous nonlinear dynamics," IEEE Transactions on Fuzzy Systems, vol. 24, no. 4, pp. 906-915, 2015.

[10] Z. Ge, F. Liu, and L. Meng, "Adaptive pid control for second order nonlinear systems," in Proceedings of the 2020 Chinese Control and Decision Conference (CCDC), pp. 2926-2931, IEEE, Hefei, China, August 2020.

[11] M. Van, "Higher-order terminal sliding mode controller for fault accommodation of lipschitz second-order nonlinear systems using fuzzy neural network," Applied Soft Computing, vol. 104, Article ID 107186, 2021.

[12] Y. Li and S. Tong, "Adaptive neural networks prescribed performance control design for switched interconnected uncertain nonlinear systems," IEEE Transactions on Neural Networks and Learning Systems, vol. 29, no. 7, pp. 3059-3068, 2017.

[13] Y. Li, X. Shao, and S. Tong, "Adaptive fuzzy prescribed performance control of nontriangular structure nonlinear systems," IEEE Transactions on Fuzzy Systems, vol. 28, no. 10, pp. 2416-2426, 2019.

[14] J. Na, "Adaptive prescribed performance control of nonlinear systems with unknown dead zone," International Journal of Adaptive Control and Signal Processing, vol. 27, no. 5, pp. 426-446, 2013.

[15] Y. Yang, C. Ge, H. Wang, X. Li, and C. Hua, "Adaptive neural network based prescribed performance control for teleoperation system under input saturation," Journal of the Franklin Institute, vol. 352, no. 5, pp. 1850-1866, 2015.

[16] S. Gao, X. Liu, Y. Jing, and G. M. Dimirovski, "A novel finitetime prescribed performance control scheme for spacecraft attitude tracking," Aerospace Science and Technology, vol. 118, Article ID 107044, 2021.

[17] S. A. Malek, M. Shahrokhi, E. Vafa, and A. Moradvandi, "Adaptive prescribed performance control of switched mimo uncertain nonlinear systems subject to unmodeled dynamics and input nonlinearities," International Journal of Robust and Nonlinear Control, vol. 28, no. 18, pp. 5981-5996, 2018.

[18] Q. Zhou, H. Li, L. Wang, and R. Lu, "Prescribed performance observer-based adaptive fuzzy control for nonstrict-feedback stochastic nonlinear systems," IEEE Transactions on Systems, Man, and Cybernetics: Systems, vol. 48, no. 10, pp. 1747-1758, 2017. 
[19] Y. Liu, X. Liu, and Y. Jing, “Adaptive fuzzy finite-time stability of uncertain nonlinear systems based on prescribed performance," Fuzzy Sets and Systems, vol. 374, pp. 23-39, 2019.

[20] X.-T. Tran and H. Oh, "Prescribed performance adaptive finite-time control for uncertain horizontal platform systems," ISA Transactions, vol. 103, pp. 122-130, 2020.

[21] F. Zhu, W. Xiang, and C. Yang, "Composite learning prescribed performance control of nonlinear systems," Complexity, vol. 2021, Article ID 9988328, 10 pages, 2021.

[22] J. Fei and C. Batur, "A novel adaptive sliding mode control with application to mems gyroscope," ISA Transactions, vol. 48, no. 1, pp. 73-78, 2009. 